\title{
Particle acceleration at, and gamma-ray emission from supernova remnants in chemically enriched media
}

\author{
Martin Pohl* \\ 1 University of Potsdam, Institute for Physics and Astronomy, University of Potsdam, D-14476 \\ Potsdam, Germany \\ 2 DESY, Platanenallee 6, D-15738 Zeuthen, Germany \\ E-mail: marpohleuni-potsdam.de
}

Maulik Bhatt (3), Anatoli Fedynitch (4), lurii Sushch, Robert Brose, Samata Das (5)

DESY, Platanenallee 6, D-15738 Zeuthen, Germany

3 also Sardar Vallabhbhai National Institute of Technology, India

4 now at Dept. of Physics, University of Alberta, Edmonton, Alberta, Canada T6G 2E1

E-mail: maulikdbhatt1996@gmail.com, afedynitchegmail.com,

iurii.Sushchedesy. de, robert.broseedesy.de, samadaseuni-potsdam. de

\section{Dominique Meyer}

5 University of Potsdam, Institute for Physics and Astronomy, D-14476 Potsdam, Germany

E-mail: domimeyer@uni-potsdam.de

\begin{abstract}
Supernova remnants often evolve in material with high abundance of heavy elements such as carbon or oxygen. Hadronic collisions in these enriched media spawn the production of secondary particles such as gamma rays, neutrinos, and secondary electrons with spectra that cannot be scaled from those calculated for pp collisions, potentially leading to erroneous results. We used Monte-Carlo event generators to calculate the differential production rate of secondary particles such as gamma rays, neutrinos, and secondary electrons for $\mathrm{H}, \mathrm{He}, \mathrm{C}$, and $\mathrm{O}$ nuclei as projectiles and as target material. The cross sections and the multiplicity spectra are separately computed for each of the 16 combinations of projectile and target. We describe characteristic effects in the form and normalization of spectra.
\end{abstract}

36th International Cosmic Ray Conference -ICRC2019-

July 24th - August 1st, 2019

Madison, WI, U.S.A.

\footnotetext{
* Speaker.
} 


\section{Introduction}

The material in $\mathrm{OB}$ associations and the wind zone of the progenitors of core-collapse supernovae can be enriched, which affects both the density of cosmic rays accelerated in the system and the production spectra of secondary particles such as gamma rays and neutrinos.

Cosmic-ray acceleration in enriched media is further supported by the recent detection of $60 \mathrm{Fe}$ at $500 \mathrm{MeV} / \mathrm{nuc}$ [1]. This unstable isotope is preferentially produced in supernovae, and its detection indicates that within less than a few million years after production in the supernova the particles were accelerated and transported to Earth.

We address the following questions:

- What are the spectra of heavy cosmic rays accelerated at supernova remnants?

- What is the gamma-ray signature of heavy elements in the GeV band, where the Fermi-LAT can measure?

- What is their gamma-ray spectrum at very high gamma-ray energies, where imaging atmospheric Cherenkov detectors, and in future CTA, operate?

- What are the spectra of secondary electrons and positrons produced by heavy cosmic-ray nuclei that may dominate the radio emission from starburst galaxies such as M82?

- What are the production spectra of neutrinos from heavy cosmic-ray nuclei in, e.g., starburst galaxies that have been speculated to be responsible of the TeV-PeV neutrinos seen with IceCube?

\section{Calculating the reaction matrices}

The spectral production rate of secondary particles can be defined as

$$
Q(E)=\frac{\mathrm{d} n}{\mathrm{~d} t \mathrm{~d} E \mathrm{~d} V}=n_{\mathrm{ism}} \int \mathrm{d} E_{\mathrm{CR}} N_{\mathrm{CR}}\left(E_{\mathrm{CR}}\right) c \beta_{\mathrm{CR}}\left(\sigma \frac{\mathrm{d} n}{\mathrm{~d} E}\right)
$$

Here $N_{\mathrm{CR}}$ is the differential density of cosmic rays, $\sigma$ is the inelastic cross section, and $d n / d E$ is the multiplicity spectrum of the secondary particle in question. The decay of secondary particles into stable final particles, for example gamma rays, neutrinos, and antiparticles, can be analytically followed with standard methods [2]). For a binned cosmic-ray spectrum and a binned spectral production rate, the integral above simplifies to a matrix operation that projects a vector, the cosmic-ray spectrum, onto another vector, the spectral production rate of the secondaries. Following the decay of the secondary can be incorporated into the matrix. Following [3, 4], we use the Monte-Carlo event generator DPMJET-III $[5,6]$ to calculate the inelastic cross section, $\sigma$, and the multiplicity spectra, $d n / d E$, for gamma rays, neutrinos, and secondary electrons and positrons. We separately consider $\mathrm{H}, \mathrm{He}, \mathrm{C}$, and $\mathrm{O}$ nuclei as projectiles and as targets, leading to 16 combinations that can be arbitrarily combined to represent a wide range of abundances for cosmic rays and the ambient medium. The statistical uncertainty is below $1 \%$. At low energies, $E_{\mathrm{CR}}<6 \mathrm{GeV}$, we use UrQMD [7] to calculate the multiplicity spectra at low energy, and between $6 \mathrm{GeV}$ and $13 \mathrm{GeV}$ we interpolate between UrQMD and DPMJET. The codes internally treat the decay of unstable secondaries, 
which substantially improves the accuracy of the spectra, as the method of Jones relies on double integrals for each decay, which can introduce substantial errors for binned spectra.

\section{The inelastic cross section}

For the same mass density, heavy elements have a much lower number density than, e.g., protons. The inelastic cross section is typically much larger though, and often the cross section is simply scaled $\propto A^{0.7}$ or similar. Figure 1 displays the inelastic cross section for combinations of hydrogen and carbon nuclei as function of the total energy per momentum of the projectile, $E_{\mathrm{CR}}$. We find that there is no simple scaling with mass number, and there is a substantial change in the energy dependence of the cross section.

Inelastic cross section

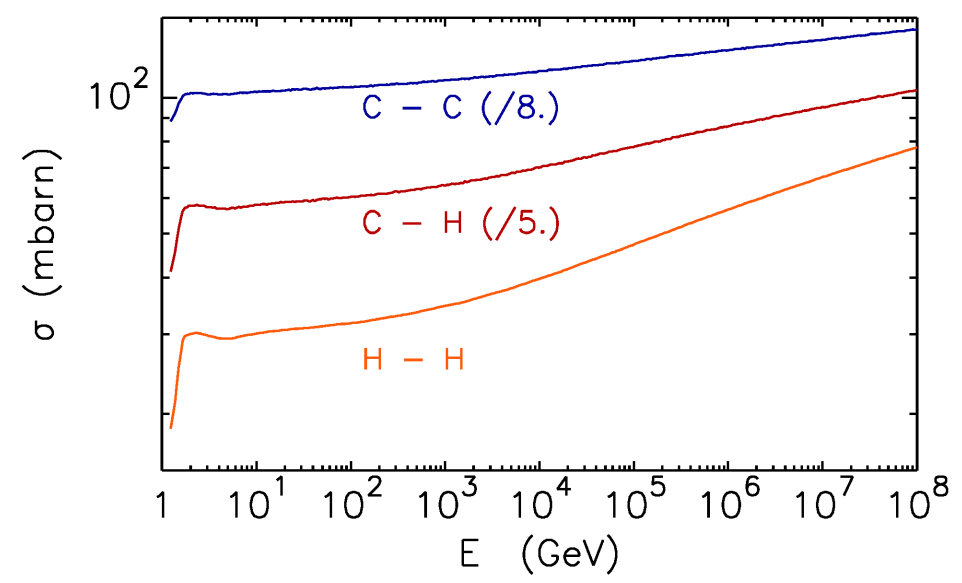

Figure 1: Inelastic cross section as function of the total energy per nucleon of the projectile. $\mathrm{C}-\mathrm{H}$ stands for carbon projectiles on a hydrogen target, and likewise for C-C and H-H. C$\mathrm{H}$ and $\mathrm{H}-\mathrm{C}$ have the same cross section.

\section{Secondary production}

\subsection{General characteristics of gamma rays}

The characteristic turnover around a $\mathrm{GeV}$ is often used to identify a hadronic origin of the emission from SNRs such as W44 and IC443. Likewise, the cut-off in the gamma-ray spectrum can be used to infere whether or not a certain SNR is a Pevatron.

We shall first describe the typical impact of heavy nuclei. For that purpose, we shall use a generic cosmic-ray spectrum that is a power law in momentum at low energies, with index $s=2.3$, but written here in kinetic energy per nucleus,

$$
N_{\mathrm{CR}}\left(E_{\mathrm{CR}}\right) \propto\left(E_{\mathrm{CR}}+A m_{p} c^{2}\right)\left(E_{\mathrm{CR}}+2 A m_{p} c^{2}\right)^{-1.65} E_{\mathrm{CR}}^{-1.65} \exp \left(-\frac{E_{\mathrm{CR}}}{Z E_{c}}\right) .
$$

The choice of cut-off energy reflects the expectations that particle transport and acceleration scales with rigidity and hence with charge number, $Z$. We find a substantial modification in the gammaray spectra below $3 \mathrm{GeV}$. Without knowledge of the composition of the cosmic rays and the target material we would not have a clear expectation of the shape of the pion bump, which would introduce substantial uncertainties in a search for, e.g., non-thermal bremsstrahlung that may become prominent below a few hundred $\mathrm{MeV}$. 

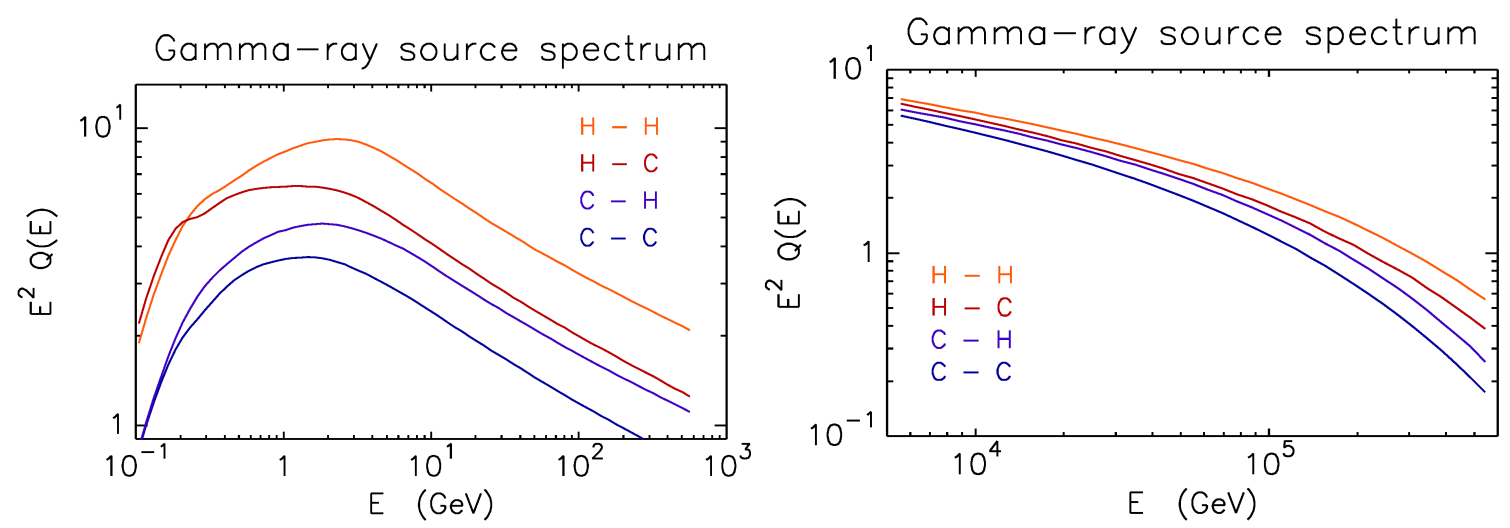

Figure 2: Arbitrarily normalized gamma-ray spectra for various compositions of cosmic rays and ambient gas. The cosmic-ray spectra follow equation 4.1. The left panel highlights the GeV band, the right panel focuses on the cut-off region.

\subsection{General characteristics of positrons}

The multiplicity spectra and charge ratio of charged pions are substantially changed for heavy elements on account of the Fermi motion of the nucleons inside the nucleus. In Figure 3 we display positron spectra that are calculated for the token cosmic-ray spectrum shown in equation 4.1, i.e. a momentum spectrum with index $\mathrm{s}=2.3$. It is evident that for heavy projectile we observe relatively few positrons below $1 \mathrm{GeV}$, and the typical kinematic turnover at $200 \mathrm{MeV}$ exists only for pp collisions.

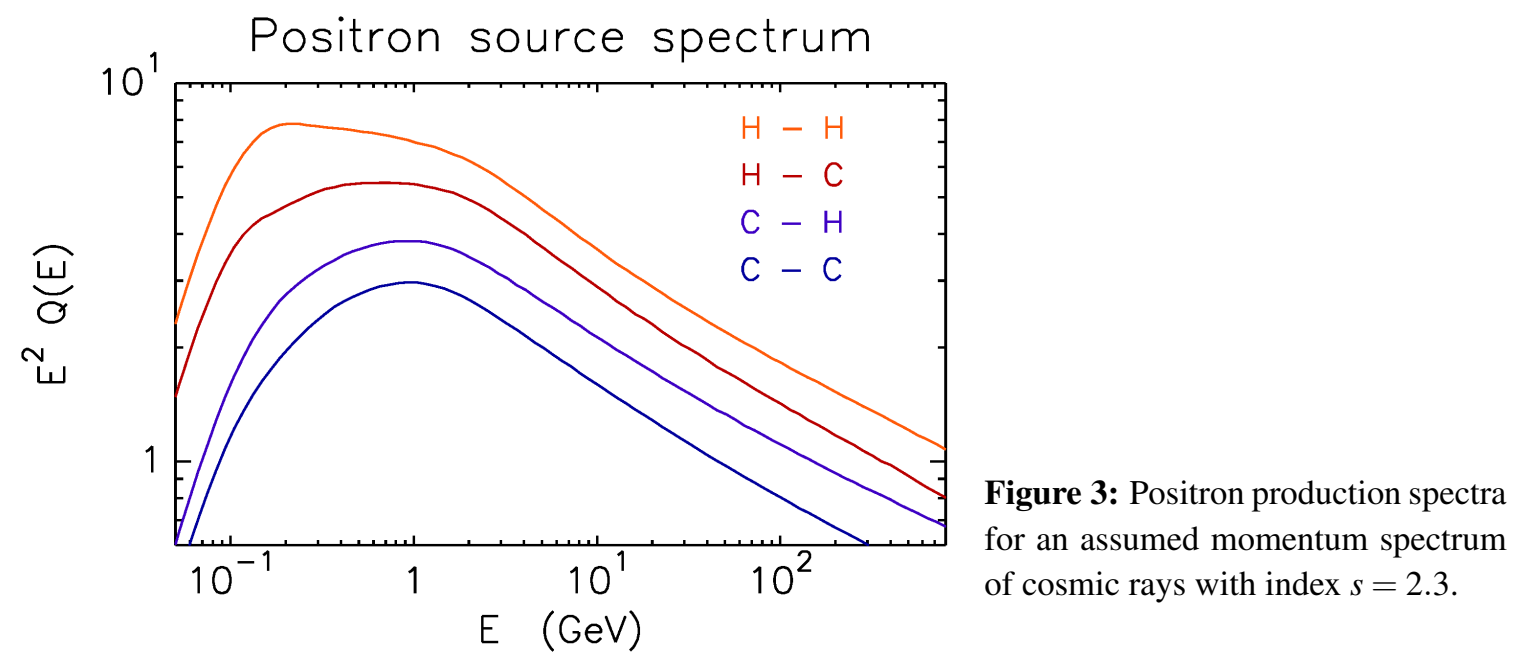

\subsection{Modelling gamma-ray emission from SNRs}

Massive stars go through various phases of evolution prior to explosion that shape their environment, and in particular the elemental abundances, in a time-dependent way [8, 9]. We developed the code RATPAC to model the acceleration and transport of cosmic rays at supernova remnants $[10,11$, and references therein], in which we simultaneously solve the transport equation for cosmic rays, the transport equation for magnetic turbulence, and the hydro-dynamical equations for the 


\begin{tabular}{|l||l|l|l|l|l|}
\hline \multicolumn{5}{|c|}{ Abundances } \\
\hline Components & ISM & RSG & WN & WO & WC \\
\hline Hydrogen & 0.71 & 0.639 & 0.20 & 0.0 & 0.0 \\
Helium & 0.28 & 0.349 & 0.78 & 0.14 & 0.55 \\
Oxygen & $2.06 \mathrm{e}-3$ & $5.41 \mathrm{e}-3$ & 0.0 & 0.24 & 0.05 \\
Carbon & $2 \mathrm{e}-3$ & $1.42 \mathrm{e}-3$ & $1 \mathrm{e}-4$ & 0.62 & 0.4 \\
Iron & $4 \mathrm{e}-4$ & $1.35 \mathrm{e}-3$ & $1.4 \mathrm{e}-3$ & 0.0 & $1.6 \mathrm{e}-3$ \\
\hline
\end{tabular}

Table 1: Mass fractions of certain elements assumed for the wind models considered here.

gas flow. Tests demonstrate that to better than $10 \%$ accuracy the cosmic-ray spectra of different elements scale in the total energy divided by the charge number, i.e. the rigidity in case of relativistic particles. The scaling in the normalization of spectra is more complex than the simple mass-number correction of the mass density in the gas fluid to the number density scaling relevant for injection into shock acceleration, because most injected particles reside at low energies near the injection energy. For the same injection energy, e.g. the same multiple of the downstream temperature, $k T$, the injection rigidity scales with the mass number and the charge number as $R \propto \sqrt{A} / Z$.

We used RATPAC to follow the an SNR evolve in a $\rho \propto 1 / r^{2}$ wind zone, for which we changed the composition, but left the mass density constant. Hence the evolutionary state of the SNR is the same for all compositions. Table 1 lists the mass fractions for the main elements that we took for our five stellar-wind models. In contrast to Figure 2, The gamma-ray spectra in Figure 4 do not arise from cosmic-ray spectra set by hand. Instead they reflect the entire acceleration history of a remnant in the Sedov phase and its internal structure. Also, the spectra are not normalized, and their amplitude indicates the reduction in numer density for heavy elements that is not fully compensated by their higher interaction cross section. To be noted from Figure 4 is that on would observe fewer gamma rays from SNR evolving in a wind zone with heavy composition (WO or WC).

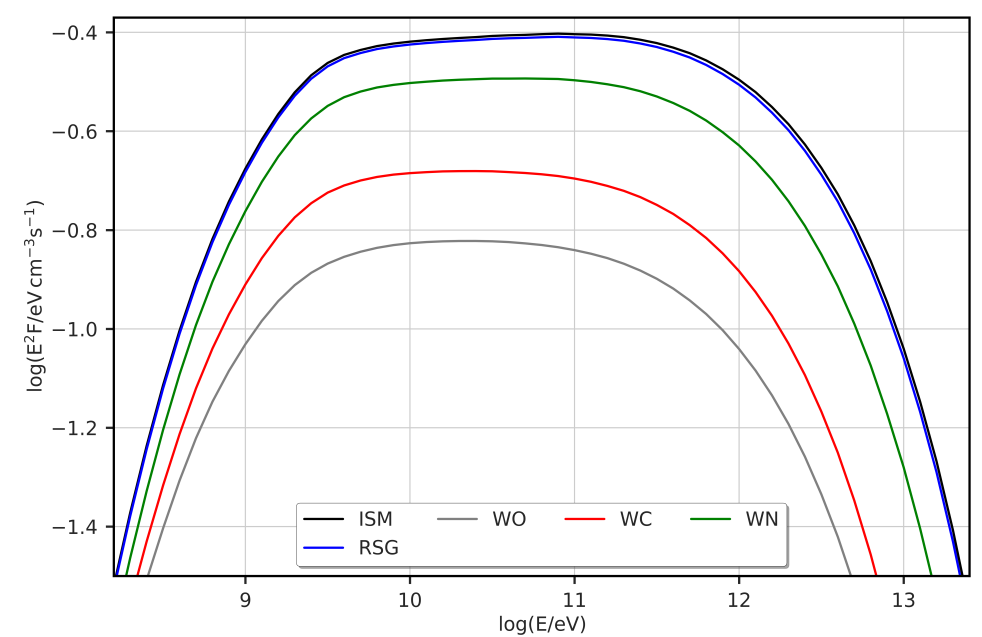

Figure 4: Gamma-ray spectra from an SNR in Sedov evolution at an age of 1000 years. The five models of wind composition are detailed in Table 1. 


\section{Conclusions}

Heavy elements among cosmic rays and in the ambient medium substantially change the spectra of gamma rays and other secondary particles that SNRs produce.

In detail we find

- The inelastic cross section does not simply scale with mass number

- Heavy cosmic rays produce relatively few positrons below $1 \mathrm{GeV}$

- The gamma-ray spectra below $3 \mathrm{GeV}$ vary depending on composition, which complicates the search for nonthermal bremsstrahlung

- The gamma-ray spectra cut-off at lower energy for a heavy composition, because the scaling with charge number of the transport and the acceleration does not fully compensate the reduction in energy per mometum, i.e. with the mass number

- We expect fewer gamma rays from SNRs evolving in a wind zone with heavy composition (WO or WC)

\section{References}

[1] Binns, W. R., Israel, M. H., Christian, E. R., et al. 2016, Science, 352, 677

[2] Jones, F. C. 1963, JGR, 68, 4399

[3] Huang, C.-Y., Park, S.-E., Pohl, M., et al. 2007, Astroparticle Physics, 27, 429

[4] Huang, C.-Y., \& Pohl, M. 2008, Astroparticle Physics, 29, 282

[5] Roesler, S., Engel, R., \& Ranft, J. 2001, International Cosmic Ray Conference, 2, 439

[6] Fedynitch, A. 2015, Cascade equations and hadronic interactions at very high energies, Ph.D. Thesis, CERN-THESIS-2015-371

[7] Bass, S. A., Belkacem, M., Bleicher, M., et al. 1998, Progress in Particle and Nuclear Physics, 41, 255

[8] Sander, A., Hamann, W.-R., \& Todt, H. 2012, A\&A, 540, A144

[9] Todt, H., Sander, A., Hainich, R., et al. 2015, A\&A, 579, A75

[10] Sushch, I., Brose, R., \& Pohl, M. 2018, A\&A, 618, A155

[11] Brose, R., Sushch, I., Pohl, M., et al. 2019, arXiv e-prints, arXiv:1906.02725 